\title{
SOX2 promoter hypermethylation in non- smoking Taiwanese adults residing in air pollution areas
}

\author{
Disline Manli Tantoh', Ming-Fang $\mathrm{Wu}^{1,2}$, Chien-Chang Ho ${ }^{3}$, Chia-Chi Lung ${ }^{1}$, Kuan-Jung Lee ${ }^{1}$, Oswald Ndi Nfor ${ }^{1}$, \\ Yi-Chia Liaw ${ }^{4}$, Shu-Yi Hsu', Pei-Hsin Chen ${ }^{1}$, Chin Lin ${ }^{5}$, Hou-Wei Chu', Yi-Ching Liaw ${ }^{7}$ and Yung-Po Liaw ${ }^{1,8^{*}}$
}

\begin{abstract}
Background: Both SOX2 promoter methylation and air pollution have been associated with lung cancer risk. However, little has been done to assess SOX2 promoter methylation in individuals living in air pollution areas. The aim of this study was to investigate SOX2 promoter methylation in non-smoking Taiwanese adults living in areas with different levels of air pollution especially particulate matter with diameter $<2.5 \mu \mathrm{m}\left(\mathrm{PM}_{2.5}\right)$.

Methods: A total of 1142 individuals aged 30-70 years were recruited. Data on SOX2 methylation, residence, age, and exposure to second-hand smoke (SHS) among others were extracted from the Taiwan Biobank dataset (2008-2015). After excluding former and current smokers, alongside those with incomplete information, a total of 461 non-smokers comprising 176 men and 285 women were included in the study. Participants' residences were grouped under northern and central/southern areas because air pollution $\left(\mathrm{PM}_{2.5}\right)$ is lower in northern compared to central and southern areas.

Results: The methylation levels in men $(0.16310 \pm 0.01230)$ and women $(0.15740 \pm 0.01240)$ were significantly different $(P<.0001)$. In both sexes, the SOX2 promoter region was shown to be significantly hypermethylated in central and southern areas compared with the northern areas. The regression coefficient $(\beta)$ was $0.00331(P=0.0257)$ in men and 0 . $00514(P<.0001)$ in women.

Conclusion: SOX2 was significantly hypermethylated in both men and women residing in central and southern areas. The consistency in the results for both sexes shows that SOX2 promoter methylation could serve as a potential biomarker for industrial air pollution exposure. Moreover, it might reflect predisposition to cancer. Hence, healthy nonsmokers at precancerous stages who have not been clinically diagnosed could be identified.
\end{abstract}

Keywords: SOX2, Hypermethylation, Non-smoking, Air pollution, Biomarker, Taiwan Biobank

\section{Background}

SOX2, also known as sex-determining region Y (SRY)-box 2 , is an oncogenic transcription factor located on chromosome 3. It maintains pluripotency or self-renewal in embryonic stem cells and has been associated with several types of cancer [1-3]. In cancers, SOX2 promoter hypermethylation has been shown to be in constant correlation

\footnotetext{
* Correspondence: Liawyp@csmu.edu.tw

${ }^{1}$ Department of Public Health and Institute of Public Health, Chung Shan Medical University, No. 110 Sec. 1 Jianguo N. Road, Taichung City 40201, Taiwan

${ }^{8}$ Department of Family and Community Medicine, Chung Shan Medical University Hospital, Taichung City, Taiwan

Full list of author information is available at the end of the article
}

with an unfavorable clinical course and poor prognosis. For example, in esophageal squamous cell carcinoma, SOX2 promoter hypermethylation seemed to have caused SOX2 silencing and the loss of SOX2 expression was associated with poor prognostic outcome [3]. In lung cancer particularly, the overexpression of SOX2 has been associated with better prognosis and survival $[2,4,5]$.

Lung cancer is one of the leading causes of global cancer mortality [6, 7]. In Taiwan, it was ranked the second incident cancer in 2014 and the top cause of mortality in 2016 [8]. Histologically, it is classified into small cell lung cancer (SCLC) and non-small cell lung cancer (NSCLC) [7]. SCLC and NSCLC respectively constitute about 15

(C) The Author(s). 2019 Open Access This article is distributed under the terms of the Creative Commons Attribution 4.0 International License (http://creativecommons.org/licenses/by/4.0/), which permits unrestricted use, distribution, and 
and $85 \%$ of lung cancer [7]. SCLC is more common in former and current smokers even though certain uncommon cases have been reported in never-smokers $[2,6,9-$ 11]. NSCLC traditionally occurs in non-smokers and comprises three subtypes: adenocarcinoma (AC), squamous cell carcinoma (SCC), and large cell carcinoma (LCC) [7].

Several environmental, lifestyle, and genetic factors have been associated with lung cancer $[6,7,11-14]$. One of such factors is air pollution resulting from increased industrialization and urbanization [14-17]. Due to industrialization, $\mathrm{PM}_{2.5}$ pollution is lower in the northern regions compared to the central and southern regions of Taiwan [16, 18-20]. The carcinogenicity of air pollutants has not been fully explained. However, it is thought that markers like DNA methylation, mutation, and polymorphism could provide some insights into the mechanisms underlying this carcinogenicity [17].

SOX2 is believed to be a potential molecular marker for lung cancer [2]. In non-smokers exposed to air pollution, SOX2 promoter methylation might help in the early prediction of lung cancer risk. However, research has not been extensively conducted as far as this domain is concerned. Therefore, this study was conducted to investigate the association between SOX2 methylation in non-smoking Taiwanese adults residing in areas with different levels of air pollution especially $\mathrm{PM}_{2.5}$.

\section{Results}

The basic characteristics of the participants are shown in Table 1. The methylation levels in men $(0.16310 \pm$ $0.01230)$ and women $(0.15740 \pm 0.01240)$ were significantly different $(P<.0001)$. The mean $( \pm \mathrm{SD})$ concentrations of $\mathrm{PM}_{2.5}\left(\mu \mathrm{g} / \mathrm{m}^{3}\right)$ from 2006 to 2011 in the northern and central/southern areas are shown in Table 2. $\mathrm{PM}_{2.5}$ concentrations in central/southern regions were higher compared to the northern regions, irrespective of the year. After adjusting for age, exposure to second-hand smoke (SHS), exercise, drinking, body fat, BMI, waist-hip ratio (WHR), asthma, and emphysema, SOX2 promoter methylation was significantly hypermethylated in both men and women residing in central and southern areas compared with the northern

Table 1 Basic characteristics of the study participants by sex

\begin{tabular}{|c|c|c|c|}
\hline Variable & Men $(n=176)$ & Women $(n=285)$ & $P$ value \\
\hline SOX2 promoter beta value & $0.1631 \pm 0.0123$ & $0.1574 \pm 0.0124$ & $<.0001$ \\
\hline Area & & & 0.5223 \\
\hline Northern & $86(48.86 \%)$ & $148(51.93 \%)$ & \\
\hline Central and southern & $90(51.14 \%)$ & $137(48.07 \%)$ & \\
\hline Age & $48.8693 \pm 11.7430$ & $48.7895 \pm 11.0194$ & 0.9413 \\
\hline Exposure to SHS & & & 0.9857 \\
\hline No & 158 (89.77\%) & $256(89.82 \%)$ & \\
\hline Yes & $18(10.23 \%)$ & $29(10.18 \%)$ & \\
\hline Exercise & & & 0.4866 \\
\hline No & $104(59.09 \%)$ & $159(55.79 \%)$ & \\
\hline Yes & $72(40.91 \%)$ & $126(44.21 \%)$ & \\
\hline Drinking & & & 0.0004 \\
\hline Never & $160(90.91 \%)$ & $281(98.60 \%)$ & \\
\hline Former & $4(2.27 \%)$ & $1(0.35 \%)$ & \\
\hline Current & $12(6.82 \%)$ & $3(1.05 \%)$ & \\
\hline Body fat (\%) & $22.5494 \pm 5.6312$ & $31.1582 \pm 6.2627$ & $<.0001$ \\
\hline BMI $\left(\mathrm{kg} / \mathrm{m}^{2}\right)$ & $24.8437 \pm 3.5941$ & $23.0715 \pm 3.3297$ & $<.0001$ \\
\hline Waist-hip ratio & $0.8848 \pm 0.0542$ & $0.827 \pm 0.0659$ & $<.0001$ \\
\hline Asthma & & & 0.8177 \\
\hline No & 167 (94.89\%) & $269(94.39 \%)$ & \\
\hline Yes & $9(5.11 \%)$ & $16(5.61 \%)$ & \\
\hline Emphysema & & & 0.3410 \\
\hline No & $173(98.30 \%)$ & $276(96.84 \%)$ & \\
\hline Yes & $3(1.70 \%)$ & 9 (3.16\%) & \\
\hline
\end{tabular}

Continuous variables, e.g., age and BMI, are expressed as mean \pm standard deviation (SD), while categorical variables, e.g., exercise and drinking, are expressed as percentages (\%) 
Table 2 Mean $\left( \pm\right.$ SD) concentrations of $\mathrm{PM}_{2.5}\left(\mu \mathrm{g} / \mathrm{m}^{3}\right)$ from 2006 to 2011 in the northern and central/southern areas

\begin{tabular}{llllllll}
\hline Area & $n$ & 2006 & 2007 & 2008 & 2009 & 2010 & 2011 \\
\hline Northern & 18 & $27.43 \pm 5.03$ & $28.69 \pm 5.39$ & $27.33 \pm 5.48$ & $25.96 \pm 5.32$ & $25.98 \pm 5.37$ & $26.61 \pm 5.30$ \\
Central/southern & 23 & $37.69 \pm 3.59$ & $38.03 \pm 4.05$ & $37.48 \pm 4.59$ & $38.44 \pm 3.67$ & $35.46 \pm 3.14$ & $36.75 \pm 4.97$
\end{tabular}

$S D$ standard deviation, $n$ number of monitoring stations

areas (Tables 3 and 4). In men residing in the central/ southern areas, the regression coefficient $(\beta)$ was 0.00331; $P=0.0257$ (Table 3). That is, the methylation level in male participants residing in central/southern areas was higher than in those residing in the northern areas and the difference was 0.00331. Similarly, in women residing in the central/southern areas, the regression coefficient $(\beta)$ was $0.00514 ; \quad P<0.0001$ (Table 4). That is, the methylation level in female participants residing in central/southern areas was higher than in those residing in the northern areas and the difference was 0.00514. Furthermore, the mean $\mathrm{PM}_{2.5}$ level (2006-2011) was significantly associated with higher SOX2 promoter methylation levels: $\beta=$ $0.00042 ; P<0.0001$ (Table 5). That is, a unit increase in $\mathrm{PM}_{2.5}$ levels was associated with $0.00042(P<0.0001)$ higher methylation levels. Analysis of $\mathrm{CpG}$ sites both in and outside the SOX2 promoter region also showed significant hypermethylation in participants residing in central/southern compared with the northern areas (Additional file 1: Tables S1-S3). In addition, analysis of the KRAS gene promoter CpG sites also showed significant hypermethylation in participants residing in the central and southern compared with the northern areas (Additional file 1: Table S4).

Table 3 Multiple linear regression showing beta coefficients of SOX2 promoter methylation in men

\begin{tabular}{lll}
\hline Variable & $\beta$ & $P$ value \\
\hline Area (northern: reference) & & \\
$\quad$ Central and southern & 0.00331 & 0.0257 \\
Age & 0.00027 & 0.0002 \\
Exposure to SHS (no: reference) & & \\
$\quad$ Yes & 0.00098 & 0.6719 \\
Exercise (no: reference) & & \\
$\quad$ Yes & -0.00189 & 0.2019 \\
Drinking (never: reference) & & \\
$\quad$ Former & -0.00361 & 0.4308 \\
$\quad$ Current & 0.00005 & 0.9853 \\
Body fat (\%) & -0.00039 & 0.1280 \\
BMl (kg/m ${ }^{2}$ ) & 0.00056 & 0.1411 \\
Waist-hip ratio & 0.01619 & 0.3230 \\
Asthma & -0.00427 & 0.1835 \\
Emphysema & 0.00021 & 0.9687 \\
\hline
\end{tabular}

\section{Discussion}

Much has not been done concerning the association between SOX2 promoter methylation and air pollution exposure. Apparently, the current study is the first to explore SOX2 promoter methylation in non-smoking Taiwanese adults based on exposure to air pollution. In both men and women living in the central and southern areas of Taiwan, SOX2 was significantly hypermethylated. The consistent hypermethylation in both sexes implies that SOX2 promoter methylation could be a potential biomarker for industrial air pollution exposure. It is always challenging to estimate individual exposure to air pollution due to the lack of validated tools. In most cases, individual exposure to air pollution is usually estimated using air quality indices from nearby monitoring stations [17]. Exposure misclassification might have been minimized in the current study as participants were stratified based on the degree of exposure to air pollution. The central and southern areas have a heavy concentration of industries compared to the northern areas $[16,19,21]$. The number 6 Naphtha Cracking Complex found in Central Taiwan is one of the biggest power plants in the world [22]. Moreover, the Formosa Petrochemical Corporation and many other industries are found in Central-Southern Taiwan [22]. These make

Table 4 Multiple linear regression showing beta coefficients of SOX2 promoter methylation in women

\begin{tabular}{lll}
\hline Variable & $\beta$ & $P$ value \\
\hline Area (northern: reference) & & \\
$\quad$ Central and southern & 0.00514 & $<.0001$ \\
Age & 0.00028 & $<.0001$ \\
Exposure to SHS (no: reference) & & 0.3697 \\
$\quad$ Yes & 0.00161 & \\
Exercise (no: reference) & & 0.3944 \\
$\quad$ Yes & -0.00100 & \\
Drinking (never: reference) & & 0.5870 \\
$\quad$ Former & -0.00513 & 0.6142 \\
$\quad$ Current & -0.00261 & 0.4164 \\
Body fat (\%) & -0.00019 & 0.5265 \\
BMl (kg/m ${ }^{2}$ ) & 0.00028 & 0.1891 \\
Waist-hip ratio & -0.01242 & 0.9830 \\
Asthma & 0.00005 & 0.9350 \\
Emphysema & -0.00025 &
\end{tabular}


Table 5 Multiple linear regression showing the association between mean $\mathrm{PM}_{2.5}\left(\mu \mathrm{g} / \mathrm{m}^{3}\right)$ from 2006 to 2011 and SOX2 promoter methylation

\begin{tabular}{lll}
\hline Variable & $\beta$ & $P$ value \\
\hline $\mathrm{PM}_{2.5}\left(\mu \mathrm{g} / \mathrm{m}^{3}\right)$ & 0.00042 & $<.0001$ \\
Sex (women: reference) & & \\
$\quad$ Men & 0.00144 & 0.4793 \\
Age & 0.00028 & $<.0001$ \\
Exposure to SHS (no: reference) & & \\
$\quad$ Yes & 0.00142 & 0.3030 \\
Exercise (no: reference) & & \\
$\quad$ Yes & -0.00145 & 0.1062 \\
Drinking (never: reference) & & \\
$\quad$ Former & -0.00319 & 0.4290 \\
$\quad$ Current & -0.00074 & 0.7543 \\
Body fat (\%) & -0.00030 & 0.0513 \\
BMl (kg/m ${ }^{2}$ ) & 0.00047 & 0.0854 \\
Waist-hip ratio & -0.00411 & 0.6063 \\
Asthma & -0.00153 & 0.4076 \\
Emphysema & -0.00073 & 0.7800 \\
\hline
\end{tabular}

the areas more prone to industrial emissions. Hence, they naturally serve as experimental fields for industrial air pollution. To minimize confounding due to smoking, only non-smokers were included in the final analysis and adjustments were made for exposure to SHS.

To combat smoking and its associated effects, e.g., non-communicable diseases, the Health Promotion Administration (HPA), Taiwan, aims to reduce smoking to $14 \%$ by 2025 compared to $20 \%$ in 2010 [23]. A decrease in smoking prevalence could subsequently lead to a decrease in smoking-related diseases. However, this does not eliminate the possibility of exposures to pollutants and their carcinogenic impacts. For example, NSCLC which is most common in non-smokers accounts for approximately $85 \%$ of lung cancer [7]. In addition, a greater percentage of known lung cancer patients in Asia are never-smokers $[11,13]$. Furthermore, smoking-related female lung cancer cases in Taiwan are few [14].

DNA methylation might help in the molecular staging of cancer as precancerous and cancerous states might be identified through this epigenetic modification [24]. This might also help to explain the mechanism for the initiation and progression of some tumors. For instance, lung cancer has been associated with epigenetic silencing [24-26]. Promoter hypermethylation is believed to be the molecular mechanism of this epigenetic gene silencing [24]. In esophageal cancer, SOX2 promoter hypermethylation was thought to be the main mechanism behind the silencing of SOX2 gene [3]. Moreover, in esophageal and hypopharyngeal cancer, the absence of
SOX2 expression was associated with poor prognosis [3, 27]. SOX2 silencing through hypermethylation might promote tumor initiation by evading cell-cycle arrest in addition to resisting apoptosis [3]. In the current study, SOX2 promoter hypermethylation was observed in individuals who resided in central and southern areas of Taiwan which have high levels of air pollution. Moreover, analysis of the KRAS gene promoter CpG sites also showed significant hypermethylation in participants residing in the central and southern compared with the northern areas. Exposure to air pollution predisposes to cancer [14-16]. SOX2 is believed to be a hallmark of lung cancer [2]. Therefore, in healthy non-smokers, SOX2 hypermethylation in air pollution areas might help to identify those at precancerous stages that have not been clinically diagnosed.

One of the limitations of this study is that the association between SOX2 promoter methylation and SOX2 gene expression was not determined. However, hypermethylation is believed to be associated with reduced gene expression [25]. Specifically, SOX2 promoter hypermethylation has been shown to be a vital epigenetic event that causes the silencing of the gene [3]. Moreover, it has been significantly associated with reduced expression of SOX2 RNA in choriocarcinoma and hydatidiform mole [28]. Another limitation of the study is that only non-cancerous individuals were included in this study. As such, the results may not reflect the methylation patterns in individuals with cancer.

\section{Conclusion}

In conclusion, significant hypermethylation of the SOX2 promoter region was observed in both non-smoking men and women living in industrial areas with well-known higher levels of air pollution. SOX2 promoter methylation might serve as a biomarker for air pollution in industrial areas. Moreover, it might reflect predisposition to cancer. Hence, healthy non-smokers at precancerous stages who have not been clinically diagnosed could be identified and monitored. Subsequently, prompt actions could be taken against such a public health issue. It is recommended that more should be done to explore the relationship between SOX2 methylation and air pollution.

\section{Methods}

\section{Data source}

Data were retrieved from the Taiwan Biobank database (2008-2015). The Taiwan Biobank was established in 2005 with the aim of integrating the genetic and medical information of about 200,000 ethnic Taiwanese with no history of cancer $[29,30]$. The enrollment of individuals in the Taiwan Biobank Project conforms to relevant regulations and guidelines. A total of 29 recruitment 
centers are distributed all over the country; each county or city possesses a minimum of one center [29]. A letter of consent was signed by each individual before data collection. Data were collected by well-trained researchers through questionnaires, physical examinations, and biochemical analyses of blood and urine samples.

\section{Data collection and study participants}

Participants' data on SOX2 promoter methylation, residence, age, smoking, exposure to SHS, exercise, drinking, body fat, BMI, WHR, asthma, and emphysema were extracted from the Taiwan Biobank dataset (20082015). Residence, age, smoking, exposure to SHS, exercise, drinking, asthma, and emphysema were self-reported while DNA methylation, body fat, BMI, and WHR were measured.

Venous blood $(9 \mathrm{ml})$ was collected into sodium citrate tubes and transported to the lab under $4{ }^{\circ} \mathrm{C}$. DNA was extracted from blood using Chemagic ${ }^{\mathrm{Tu}}$ Prime $^{\mathrm{mm}}$ instrument which is an automated chemical extraction machine that uses magnetized rods to separate nucleic acids from solutions. The DNA length was determined with the Fragment Analyzer (Agilent) while the purity was assessed using the optical density (OD) at 260/280. Samples with an OD 260/280 ratio of 1.6-2.0 were considered to be pure. Pure samples intended for long-term use were stored at $-80^{\circ} \mathrm{C}$. DNA samples were subjected to sodium bisulfite treatment using the EZ DNA Methylation Kit (Zymo Research, CA, USA). DNA methylation at each $\mathrm{CpG}$ site was determined with the Infinium ${ }^{\circ}$ MethylationEPIC BeadChipEPIC array (Illumina Inc.) which covers 850,000 methylation sites. Details on this platform are described elsewhere [31-33]. The methylation levels were expressed as beta-values $(\beta)$ which range between 0 and 1 . $\beta$-values were determined using the formula $\beta=M /(M+U)$, where $M$ is the methylated intensity and $U$ is the unmethylated intensity. Quality control was done according to the Illumina ${ }^{\circ}$ GenomeStudio $^{\circ}$ Methylation Module v1.8 [34]. Samples with detection $P$ value $>0.05$ and bead counts $<3$ were eliminated. Dye-bias across batches was adjusted by normalization, and background correction was performed. Outliers were removed using the median absolute deviation method.

Participants were considered to be from an area if they lived there for at least 3 months. Where participants lived was considered to be where they were likely to be exposed to air pollution. The residences were grouped into northern and central/southern regions. This is because $\mathrm{PM}_{2.5}$ pollution in the northern areas is lower compared to the central and southern areas [16, 18-20]. The northern areas included Taipei and New Taipei Cities, while the central/southern areas included Taichung and Nantou Cities, Changhua and Yunlin Counties,
Chiayi County, Tainan, and Chiayi Cities. $\mathrm{PM}_{2.5}$ statistics in these areas were obtained from the Air Quality Monitoring Database (AQMD) set up by the Environmental Protection Administration, Taiwan. There were 41 air quality monitoring stations in the study areas. Of these stations, 18 were located in the northern region while 23 were located in the central/southern regions. Annual $\mathrm{PM}_{2.5}$ readings (2006-2011) from the various stations were used to determine the annual average concentration for each region.

Participants were considered as (1) non-smokers: never smoked or did not continuously smoke for 6 months or more; (2) former smokers: continuously smoked for at least 6 months but were currently not smoking; (3) current smokers: continuously smoked for 6 months or more and were currently smoking; (4) non-drinkers: no history of alcohol drinking or weekly drinking of less than $150 \mathrm{cc}$ of alcohol for continuously 6 months; (5) former drinkers: abstained from drinking for over 6 months; (6) current drinkers: weekly drinking of at least $150 \mathrm{cc}$ of alcohol continuously for 6 months; (7) physically active: exercised for $>150$ min per week; and (8) exposed to SHS: exposure for at least $5 \mathrm{~min}$ per hour.

After excluding former and current smokers as well as those with incomplete information, a total of 461 non-smokers comprising 176 men and 285 women were included in our study. A total of 24 SOX2 CpG sites located on SOX2 promoter region were available in the Taiwan Biobank dataset. These sites were cg24513480, cg05664581, cg19258425, cg18148179, cg07747133, cg24782772, cg00666105, cg04948892, cg15106134, cg02573703, cg20106776, cg11129008, cg08062338, cg12930100, cg01023203, cg22530053, cg27331851, cg14783675, cg01340005, cg17051733, cg11142406, cg09530873, cg25933341, and cg08464053. The $\beta$-values at all the 24 SOX2 CpG sites were summed up, and the mean value was determined. In order to reach the conclusion that SOX2 is a reliable biomarker of cancer risk in air pollution areas, the mean $\beta$-value of $\mathrm{CpG}$ sites at the promoter of KRAS, another lung cancer risk gene, was determined. Ethical approval for this study was obtained from Chung Shan Medical University Institutional Review Board (CS2-17070).

\section{Statistical analysis}

Data management and analyses were performed with the SAS 9.4 software (SAS Institute, Cary, NC). Continuous variables were analyzed using $t$ test and expressed as mean \pm standard deviation (SD) while categorical variables were analyzed using chi-square test and reported as percentages (\%). Methylation data were normalized using the Illumina ${ }^{\circ}$ GenomeStudio V2011.1 software [34]. The correction of cell-type heterogeneity 
was done with the $\mathrm{R}$ software using the Reference-Free Adjustment for Cell-Type composition (ReFACTor) method [35].

The association between SOX2 promoter methylation and residential area was determined using multiple linear regression. Multivariate adjustments were performed for age, exposure to SHS, exercise, drinking, body fat, BMI, WHR, asthma, emphysema, and cell-type composition. $P$ values less than 0.05 were considered to be statistically significant.

\section{Additional file}

Additional file 1: Table S1. Multiple linear regression showing beta coefficients of SOX2 methylation both in and outside the promoter region. Table S2. Multiple linear regression showing beta coefficients of SOX2 methylation in the exon. Table S3. Multiple linear regression showing beta coefficients of SOX2 methylation in the 3UTR region. Table S4. Multiple linear regression showing beta coefficients of KRAS promoter methylation. (DOCX $22 \mathrm{~kb}$ )

\section{Abbreviations}

AC: Adenocarcinoma; BMI: Body mass index; CpG: Cytosine-phosphateguanine; DNA: Deoxyribonucleic acid; HDL: High-density lipoprotein; LCC: Large cell carcinoma; LDL: Low-density lipoprotein; MOST: Ministry of Science and Technology; $n$ : Sample size; NSCLC: Non-small cell lung cancer; SCC: Squamous cell carcinoma; SCLC: Small cell lung cancer; SD: Standard deviation; SHS: Second-hand smoke; SRY: Sex-determining region Y; TC: Total cholesterol; TG: Triglyceride; WHR: Waist-hip ratio; $\beta$ : Regression coefficient

\section{Acknowledgements}

Not applicable.

\section{Funding}

This work was funded by the Ministry of Science and Technology, MOST (MOST 105-2627-M-040-002; 106-2627-M-040-002; 107-2627-M-040-002 and 106 -EPA EPA-F-016-001)

\section{Availability of data and materials}

The data that support the findings of this study are available from Taiwan Biobank but restrictions apply to the availability of these data, which were used under license for the current study, and so are not publicly available. Data are however available from the authors upon reasonable request and with permission of Taiwan Biobank.

\section{Authors' contributions}

DMT and YPL conceived and designed the study. DMT, MFW, CCH, ONN, $Y C L, H W C$, and $Y C L$ performed the literature search. $Y P L, K L, S Y H, P H C, C L$, and $C C L$ acquired the data and performed the data analysis. DMT, MFW, $\mathrm{CCH}, \mathrm{CCL}, \mathrm{KL}$, ONN, YCL, SYH, PHC, CL, HWC, YCL, and YPL interpreted the data. DMT drafted the manuscript. DMT, ONN, and YPL made critical revisions of the manuscript for important intellectual contents. All authors approved the final version of the manuscript.

\section{Ethics approval and consent to participate}

This study was approved by the Chung Shan Medical University Institutional Review Board (CS2-17070).

\section{Consent for publication}

Not applicable.

\section{Competing interests}

The authors declare that they have no competing interests.

\section{Publisher's Note}

Springer Nature remains neutral with regard to jurisdictional claims in published maps and institutional affiliations.

\section{Author details}

${ }^{1}$ Department of Public Health and Institute of Public Health, Chung Shan Medical University, No. 110 Sec. 1 Jianguo N. Road, Taichung City 40201, Taiwan. ${ }^{2}$ School of Medicine, Chung Shan Medical University, Taichung City, Taiwan. ${ }^{3}$ Department of Physical Education, Fu-Jen Catholic University, New Taipei City, Taiwan. ${ }^{4}$ Department of Medical Education, Taipei Veterans General Hospital, Taipei, Taiwan. ${ }^{5}$ Graduate Institute of Life Sciences, National Defense Medical Center, Taipei, Taiwan. ${ }^{6}$ Institute of Biomedical Sciences, Academia Sinica, Taipei, Taiwan. ${ }^{7}$ Institute of Clinical Medicine, National Yang-Ming University, Taipei, Taiwan. ${ }^{8}$ Department of Family and Community Medicine, Chung Shan Medical University Hospital, Taichung City, Taiwan.

Received: 9 October 2018 Accepted: 3 March 2019

Published online: 12 March 2019

\section{References}

1. Santini R, Pietrobono S, Pandolfi S, Montagnani V, D'amico M, Penachioni J, Vinci M, Borgognoni L, Stecca B. SOX2 regulates self-renewal and tumorigenicity of human melanoma-initiating cells. Oncogene. 2014;33:4697.

2. Ying J, Shi C, Li CS, Hu LP, Zhang WD. Expression and significance of SOX2 in non-small cell lung carcinoma. Oncol Lett. 2016;12:3195-8.

3. Maehara R, Fujikura K, Takeuchi K, Akita M, Abe-Suzuki S, Karbanová J, Corbeil D, Itoh T, Kakeji Y, Zen Y. SOX2-silenced squamous cell carcinoma: a highly malignant form of esophageal cancer with SOX2 promoter hypermethylation. Mod Pathol. 2018;31:83.

4. Velcheti V, Schalper K, Yao X, Cheng H, Kocoglu M, Dhodapkar K, Deng Y, Gettinger S, Rimm DL. High SOX2 levels predict better outcome in nonsmall cell lung carcinomas. PLoS One. 2013;8:e61427.

5. Wilbertz T, Wagner P, Petersen K, Stiedl A-C, Scheble VJ, Maier S, Reischl M, Mikut R, Altorki NK, Moch H. SOX2 gene amplification and protein overexpression are associated with better outcome in squamous cell lung cancer. Mod Pathol. 2011;24:944.

6. Pallis AG, Syrigos KN. Lung cancer in never smokers: disease characteristics and risk factors. Crit Rev Oncol Hematol. 2013;88:494-503.

7. Pikor LA, Ramnarine VR, Lam S, Lam WL. Genetic alterations defining NSCLC subtypes and their therapeutic implications. Lung Cancer. 2013;82:179-89.

8. Health Promotion Administration MoHaW. 2017 Annual Report of Health Promotion Administration, 2017. Available at http://www.hpa.gov.tw/. Accessed 9 May 2018

9. Govindan R, Ding L, Griffith M, Subramanian J, Dees ND, Kanchi KL, Maher CA, Fulton R, Fulton L, Wallis J. Genomic landscape of non-small cell lung cancer in smokers and never-smokers. Cell. 2012;150:1121-34.

10. van Meerbeeck JP, Fennell DA, De Ruysscher DK. Small-cell lung cancer. Lancet. 2011:378:1741-55.

11. Torres-Durán M, Ruano-Ravina A, Kelsey KT, Parente-Lamelas I, Provencio M, Leiro-Fernández V, Abal-Arca J, Montero-Martínez C, Vidal-Garcia I, Pena C. Small cell lung cancer in never-smokers. Eur Respir J 2015:ERJ-01524-2015.

12. Couraud S, Zalcman G, Milleron B, Morin F, Souquet P-J. Lung cancer in never smokers-a review. Eur J Cancer. 2012:48:1299-311.

13. Subramanian J, Govindan R. Lung cancer in never smokers: a review. J Clin Oncol. 2007;25:561-70

14. Chiu H-F, Cheng M-H, Tsai S-S, Wu T-N, Kuo H-W, Yang C-Y. Outdoor air pollution and female lung cancer in Taiwan. Inhal Toxicol. 2006;18:1025-31.

15. Raaschou-Nielsen O, Andersen ZJ, Beelen R, Samoli E, Stafoggia M, Weinmayr G, Hoffmann B, Fischer P, Nieuwenhuijsen MJ, Brunekreef B. Air pollution and lung cancer incidence in 17 European cohorts: prospective analyses from the European Study of Cohorts for Air Pollution Effects (ESCAPE). Lancet Oncol. 2013;14:813-22.

16. Lo W-C, Shie R-H, Chan C-C, Lin H-H. Burden of disease attributable to ambient fine particulate matter exposure in Taiwan. J Formos Med Assoc. 2017;116:32-40.

17. Shahadin MS, Ab Mutalib NS, Latif MT, Catherine GM, Tidi H. Challenges and future direction of molecular research in air pollution-related lung cancers. Lung Cancer. 2018;118:69-75.

18. Yeh H-L, Hsu S-W, Chang Y-C, Chan T-C, Tsou H-C, Chang Y-C, Chiang P-H. Spatial analysis of ambient PM2. 5 exposure and bladder cancer mortality in Taiwan. Int J Environ Res Public Health. 2017;14:508. 
19. Chiang P, Chen CW, Hsieh DP, Chan T-C, Chiang H-C, Wen C-P. Lung cancer risk in females due to exposures to PM2. 5 in Taiwan. Open Epidemiol J. 2014;7:6-16.

20. Chen M-L, Mao I-F, Lin I-K. The PM2. 5 and PM10 particles in urban areas of Taiwan. Sci Total Environ. 1999;226:227-35.

21. Chio C-P, Liao C-M, Tsai Y-I, Cheng M-T, Chou W-C. Health risk assessment for residents exposed to atmospheric diesel exhaust particles in southern region of Taiwan. Atmos Environ. 2014;85:64-72.

22. Yuan T-H, Shie R-H, Chin Y-Y, Chan C-C. Assessment of the levels of urinary 1-hydroxypyrene and air polycyclic aromatic hydrocarbon in PM2. 5 for adult exposure to the petrochemical complex emissions. Environ Res. 2015;136:219-26.

23. Health Promotion Administration MoHaW, ROC (Taiwan). 2016 Taiwan tobacco control annual report Taiwan, 2017. Available at http://tobacco.hpa.gov.tw. Accessed 9 May 2018.

24. Brock MV, Hooker CM, Ota-Machida E, Han Y, Guo M, Ames S, Glöckner S, Piantadosi S, Gabrielson E, Pridham G. DNA methylation markers and early recurrence in stage I lung cancer. N Engl J Med. 2008;358:1118-28.

25. Herman JG, Baylin SB. Gene silencing in cancer in association with promoter hypermethylation. N Engl J Med. 2003;349:2042-54.

26. Baylin SB, Ohm JE. Epigenetic gene silencing in cancer-a mechanism for early oncogenic pathway addiction? Nat Rev Cancer. 2006;6:107.

27. Avincsal MO, Jimbo N, Fujikura K, Shinomiya H, Otsuki N, Morimoto K, Furukawa T, Morita N, Maehara R, Itoh T. Epigenetic down-regulation of SOX 2 is an independent poor prognostic factor for hypopharyngeal cancers. Histopathology. 2018;72:826-37

28. Li AS, Siu MK, Zhang H, Wong ES, Chan KY, Ngan HY, Cheung AN. Hypermethylation of SOX2 gene in hydatidiform mole and choriocarcinoma. Reprod Sci. 2008;15(7):735-44.

29. Purpose Of Taiwan Biobank [https://www.twbiobank.org.tw/new_web_en/ index.php].

30. Fan C-T, Lin J-C, Lee C-H. Taiwan Biobank: a project aiming to aid Taiwan's transition into a biomedical island. Pharmacogenomics. 2008;9(2):235-46.

31. Bojovic B, Blancher C. Epigenetic analysis on Illumina EPIC arrays. Epigenetics. 2017;28:03.

32. Gujar H, Liang JW, Wong NC, Mozhui K. Profiling DNA methylation differences between inbred mouse strains on the Illumina Human Infinium MethylationEPIC microarray. PLoS One. 2018;13(3):e0193496.

33. Infinium assay lab setup and procedures guide [https://support.illumina. com/downloads/infinium-assay-lab-setup-and-procedures-11322460.html].

34. Module, GenomeStudio Methylation v1. 8 User Guide [https:/support illumina.com/content/dam/illumina-support/documents/documentation/ software_documentation/genomestudio/genomestudio-2011-1/ genomestudio-methylation-v1-8-user-guide-11319130-b.pdf].

35. Rahmani E, Zaitlen N, Baran Y, Eng C, Hu D, Galanter J, Oh S, Burchard EG, Eskin E, Zou J. Sparse PCA corrects for cell type heterogeneity in epigenome-wide association studies. Nat Methods. 2016;13(5):443.

Ready to submit your research? Choose BMC and benefit from:

- fast, convenient online submission

- thorough peer review by experienced researchers in your field

- rapid publication on acceptance

- support for research data, including large and complex data types

- gold Open Access which fosters wider collaboration and increased citations

- maximum visibility for your research: over $100 \mathrm{M}$ website views per year

At $\mathrm{BMC}$, research is always in progress.

Learn more biomedcentral.com/submissions 